durchschnittlich ein bis zwei Jahren, die in der Regel auch noch zur Bewährung ausgesetzt werden. ${ }^{60}$ Solche Strafen sind nicht dazu angetan, eine „abschreckende und damit präventive Wirkung" zu entfalten, wie die Europäische Kommission bereits in ihrem ersten Fortschrittsbericht nach dem EU-Beitritt Rumäniens im Juni 2007 zu Recht kritisierte. ${ }^{61}$ Es ist nicht zu erwarten, dass sich diese Rechtsprechungspraxis in naher Zukunft grundlegend ändern wird.

Fünftens schließlich besteht für all diejenigen korruptionsverdächtigen Politiker, die aktuell als Parlamentarier tätig sind, ein weiterer Schutzmechanismus: ihre parlamentarische Immunität. Wie eingangs bereits erwähnt, dürfen sie ohne die Zustimmung ihrer Kammer „nicht durchsucht, festgehalten oder verhaftet werden“ (Art. 72 Abs. 1 Verf.). Ein eventueller Vollzug von Gefängnisstrafen läge also erneut in der politischen Entscheidungsbefugnis des Parlaments, und es dürfte interessant sein zu sehen, ob die Legislative tatsächlich einer Inhaftierung prominenter Parlamentarier zustimmen würde. Gegenwärtig genießen vier der ehemaligen Regierungsmitglieder diesen Schutz: die Abgeordneten Tudor Chiuariu, Monica Iacob-Ridzi und Adrian Năstase sowie der Senator Miron Mitrea. Zu diesen ehe-maligen Premierministern beziehungsweise Ministern kommen derzeit acht (!) weitere Mitglieder des rumänischen Parlaments hinzu, gegen die wegen anderweitiger Korruptionsvorwürfe ermittelt wird. Die Tageszeitung Cotidianul witzelte daher nach den Parlamentswahlen vom November 2008: „Antikorruptionsdirektion hat eigene Parlamentsfraktion“62. Ob die Bekämpfung der „großen“ politischen Korruption in Rumänien daher in nächster Zeit tatsächlich erste greifbare Erfolge zeitigen wird, muss weiterhin skeptisch eingeschätzt werden.

60 Der Fall Cutean ist bisher eine seltene Ausnahme und zudem noch nicht letztinstanzlich entschieden.

61 Kommission der Europäischen Gemeinschaften, Bericht über Rumäniens Fortschritte bei den Begleitmaßnahmen nach dem Beitritt, Brüssel, 27. Juni 2007, S. 19, http://ec.europa.eu/dgs/secretariat_general/cvm/docs/romania_report_20070627_de.pdf (Abruf am 9. März 2009). Allerdings drängt sich beispielsweise aus bundesdeutscher Perspektive die Frage auf, ob hinsichtlich der Strafmaße tatsächlich ein Unterschied zu den „konsolidierten“ Rechtsstaaten Mittel- und Westeuropas besteht, wie es der Fortschrittsbericht impliziert.

62 Cotidianul vom 2. Dezember 2008, http://www.cotidianul.ro/dna_are_grup_parlamentar-66471. html (Abruf am 15. März 2009). Einer dieser acht Parlamentarier, der neu gewählte Abgeordnete Virgil Pop (PNL), befand sich zum Zeitpunkt der Wahlen sogar in Untersuchungshaft.

\title{
Das zyprische Repräsentantenhaus zwischen Verfassungsvorgaben und Teilungsnöten: Entwicklungen seit 1960
}

\section{Dimitrios Parashu}

Die Republik Zypern, seit dem 1. Mai 2004 eines der jüngeren Mitglieder der Europäischen Union, hat nicht erst seit ihrer Unabhängigkeit vom Vereinigten Königreich 1960 eine stürmische Geschichte hinter sich. Man weiß, dass die Insel seit dem Sommer 1974 de facto in zwei geographische Hälften geteilt ist: Den (international als Republik Zypern anerkannten) griechischen Süden und den (nur von Ankara als eigenständige „Republik“ ge- 
sehenen) türkischen Norden. An der Wiege dieser Division stand die aufgrund der Art ihrer bikommunalen Anlage schwer umsetzbare Verfassung von $1960^{1}$, von der im Folgenden noch die Rede sein wird; schon ab 1963, als Staatspräsident Makarios III. seinen 13-Punkte-Vorschlag hinsichtlich einer sicherlich nötigen Verfassungsänderung vorstellte ${ }^{2}$, und den ethnischen Unruhen der Folgezeit nahmen die türkischen Zyprer an den verfassungsrechtlich vorgesehenen Organen und Prozeduren der Insel nicht mehr teil. Seither sind einige Versuche fehlgeschlagen, die faktische Teilung der einzigen reinen Präsidialrepublik innerhalb der EU zu überwinden. Aktuell haben sich entsprechende Bemühungen wieder verstärkt ${ }^{3}$; es bleibt abzuwarten, ob diese von Erfolg gekrönt sein werden. Ein Verfassungsorgan, das sehr stark von der Teilung der Insel betroffen ist, ist das zyprische Repräsentantenhaus (Vouli ton Antiprosopon / Temsilciler Meclisi) ${ }^{4}$. Wegen der EU-Mitgliedschaft Zyperns und im Sinne eines Zusammenwachsens nicht nur Europas sollen einige bisher wohl unbekannte Fakten und Problematiken hinsichtlich seiner Entwicklung und Eigenheit vorgestellt werden.

\section{Institutionelle Problematiken des Repräsentantenhauses}

Die im Jahre der Unabhängigkeit (1960) verabschiedete Verfassung der Republik Zypern sieht in ihren Art. 61 bis $85^{5}$ Näheres betreffend des Hohen Hauses der Insel vor ${ }^{6}$. Die

1 Die Verfassung von 1960 war das Produkt der Züricher und Londoner Gespräche zwischen Griechen, Türken und Briten und als solches dem zyprischen Volk quasi ,auferlegt" worden (da der britische Einfluss auf das vierköpfige verfassungsschaffende Komitee faktisch unübersehbar war; vgl. Kriton Tornaritis, Das Staatsrecht der Republik Zypern (auf Griechisch), Nikosia 1982, S. 16 - 18). Den Zyprern selbst wurde nicht die Möglichkeit gegeben, per Referendum darüber abzustimmen. Dieses Verfassungswerk ist von verschiedenen Rechtswissenschaftlern als „umständlich“ charakterisiert worden und hat seinen Teil dazu beigetragen, das Zypernproblem zu vergrößern - insbesondere durch die Überbetonung der Verschiedenheiten von Zyperngriechen und -türken; vgl. dazu unter anderem ebenda, S. 16 - 18; Maria Giannakaki, Makarios (auf Griechisch), Athen 2004, S. 25 - 27.

2 Vgl. Konstantinos Goumas / Stelios Koutsiopoulos (Hrsg.), Chronik des 20. Jahrhunderts (auf Griechisch), Athen 1990, S. 980; Panagiotis Lambrias, Karamanlis, der Freund (auf Griechisch), Athen 1998, S. 309; Iossif Iossif, Zypernproblem und Internationale Politik (auf Griechisch), Athen 2000, S. 214; Maria Giannakaki, a.a.O. (Fn. 1), S. 30 f.

3 Vgl. den entsprechenden Artikel der Welt Online vom 21. März 2008, Neue Hoffnung auf Wiedervereinigung Zyperns, http://www.welt.de/politik/article1824815/Neue_Hoffnung_auf_Wiedervereinigung_Zyperns.html (Abruf am 21. März 2008); ebenso den relevanten Artikel der Zeitung Kathimerini vom 2. Dezember 2008 (auf Griechisch).

4 Vgl. http://www.parliament.cy/www_START7index.asp; www.parliament.cy/parliamentgr/home. htm (auf Griechisch) (Abruf am 25. März 2009); Dimitrios Parashu, Resultate von Parlamentswahlen der Mitgliedsländer der Europäischen Union seit 1945, Kapitel 17: Zypern (auf Griechisch; unveröffentlicht).

5 Gisbert Flanz / Rüdiger Wolfrum / Rainer Grote, Constitution of Cyprus, in: Max-Planck-Institut für ausländisches öffentliches Recht und Völkerrecht (Hrsg.), Constitutions of the Countries of the World, New York 2005, S. 295 ff.

6 Es wird schon in Art. 61 fundamental klargestellt, dass das Hohe Haus all jene gesetzgeberischen Kompetenzen für sich in Anspruch nehmen kann, die nicht ausdrücklich den kommunalen Kammern der griechischen und der türkischen Zyprer vorbehalten sind; vgl. betreffend letztgenannter Kammern die Artikel 86 ff. der Verfassung, wobei insbesondere Fragen der, naturgemäß verschie- 
Teilung und die Abwesenheit der türkischen Zyprer aus den Verfassungsorganen aber brachten bereits ab 1963 faktische Schwierigkeiten der Umsetzung des Verfassungswerks mit sich. Als praktisch besonders akute Beispiele seien hier die folgenden angeführt: (1) In Art. 62 wird festgelegt, dass die Anzahl der Abgeordneten 50 zu betragen hat, unter Vorbehalt eines diese Zahl erhöhenden Beschlusses durch das Parlament selbst; ein solcher Beschluss müsste allerdings von einer Zweidrittelmehrheit sowohl der griechisch- als auch der türkisch-zyprischen Abgeordneten getragen werden (Art. 62 Abs.1). (2) Als ebenso problematisch sollte sich in der Praxis Art. 182 Abs. $3^{7}$ erweisen, der für eine Verfassungsrevision jeweils mindestens Zweidrittelmehrheiten der griechisch- und türkisch-zyprischen Mitglieder des Repräsentantenhauses vorschreibt. Diese Regelung hat bisher auch die Umsetzung von anderen Verfassungsartikeln maßgeblich beeinflusst, insbesondere die für Parlamentswahlen wichtigen Art. 63 und 66. (3) Das Quorum zur Eröffnung und Legitimation des Wirkens des Repräsentantenhauses, auf mindestens ein Drittel aller gesetzlichen Mitglieder festgelegt (Art. 77), wird (trotz der Nichtteilnahme der 24 türkisch-zyprischen Abgeordneten) stets problemlos eingehalten. (4) Ebenso wichtig ist in diesem Zusammenhang der Art. 78 Abs. 1, der für die Abstimmung über Gesetze und Beschlüsse des Hohen Hauses die einfache Mehrheit der anwesenden Mandatsträger verlangt; Abs. $2^{8}$ sieht diese Mehrheiten sowohl für die griechisch- als auch die türkisch-zyprischen Abgeordneten vor, wann immer ein Gesetz über die Kommunen, das Steuerrecht oder eine Wahlgesetzänderung ansteht. Diese Regelung hatte in der Praxis des Öfteren nahe liegende Probleme ${ }^{9}$ und im Regelfall die Beschließung solcher Gesetze durch die griechisch-zyprischen Abgeordneten auf der sogleich vorzustellenden Basis des Notrechts zur Folge.

denen, Religionen und des Bildungswesens, aber auch Facetten des Personensteuerrechts und, wie etwa im türkisch-zyprischen Fall „Murveddet Toygar vs. Ali Ratib“ (1963), 2 CLR 312, des Familien- und Erbrechts eine gewichtige Rolle spielen. Dieses ist seit der Teilung 1974 dahingehend verändert, dass das Repräsentantenhaus nunmehr auch diese legislativen KommunalkammerKompetenzen für die Republik Zypern wahrnimmt, de facto allerdings schon früher: Bereits nach den angesprochenen Unruhen hatte sich die griechisch-zyprische Kommunalkammer im März 1965 aufgelöst und ihre gesetzgeberischen Kompetenzen dem Hohen Hause zuerkannt, vgl. Konstantinos Goumas / Stelios Koutsiopoulos (Hrsg.), a.a.O. (Fn. 2), S. 997 ff.

7 Art. 182 Abs. 1 schließt übrigens explizit Teile der Verfassung von einer Revision aus (so den Annex-Teil III, ein Produkt des Züricher Vertrages vom 11. Februar 1959).

8 Hinsichtlich des Problems von Art. 78 Abs. 2 vgl. Jörg-Detlef Kühne, Der zyprische Verfassungskonflikt. Eine kritische Würdigung der Lösungsvorschläge, in: Beiträge zur Konfliktforschung, 3. Jg. (1973), S. $71-87$, S. 75.

9 Beispielsweise beschloss die erforderliche Mehrheit der türkisch-zyprischen Abgeordneten am 18. Dezember 1962 im Gegensatz zur entsprechenden Mehrheit ihrer griechisch-zyprischen Kollegen, das seinerzeit geplante Einkommensteuergesetz nicht zu verabschieden, vgl. Konstantinos Goumas / Stelios Kotsiopoulos (Hrsg.), a.a.O. (Fn. 2), S. 966; darüber hinaus ist auch von Interesse, dass die türkisch-zyprischen Abgeordneten in den Jahren 1960 bis 1963 insgesamt drei Mal (vgl. Achilleus Aimilianidis, Die Überwindung der Zyprischen Verfassung (auf Griechisch), Athen / Thessaloniki 2006, S. 67), nach entsprechendem Rückverweis eines vom Hohen Haus einstimmig beschlossenen Gesetzes durch Vizestaatspräsident Fazil Kücük, ihr Votum änderten und einige Gesetze, wie etwa dasjenige über die Rehabilitierung öffentlicher Angestellter, die durch die britische Kolonialregierung freigestellt worden waren (1961), letztendlich nicht zustande kamen; vgl. Republik Zypern, Sitzungsprotokolle des Repräsentantenhauses (16. August 1961 bis 31. Dezember 1961), Sitzung vom 2. November 1961, S. 519 - 520 (auf Griechisch). 
Für die Binnenorganisation des Repräsentantenhauses legt Art. 72 Abs. 1 fest, dass der Präsident des Hohen Hauses ein Zyperngrieche zu sein hat, gewählt von den griechischzyprischen Volksvertretern. Vizepräsident soll ein Zyperntürke sein, den die türkisch-zyprischen Mandatsträger zu wählen haben. Jedoch ist seit dem Rückzug der Zyperntürken aus allen öffentlichen Ämtern auch das Amt des Parlamentsvizepräsidenten seit 1963 vakant. Der Verfassungsgeber von 1960 hatte für den Fall der Vakanz eines der beiden Hauptämter des Parlamentspräsidiums in Abs. 2 desselben Artikels eine „schnellstmögliche Wahl“ zur Besetzung dieser Position vorgeschrieben - naturgemäß konnte (oder wollte) er die dann relativ raschen Entwicklungen nicht in vollem Maß vorhersehen. Die Parlamentspraxis der Insel sieht heute so aus, dass allein der Präsident des Repräsentantenhauses die Kompetenzen des Präsidiums wahrnimmt. Bei nur temporärer Vakanz dieser Posten soll verfassungsgemäß der jeweils älteste Abgeordnete der Griechen und Türken das Amt ausüben (Art. 72 Abs. 3), wie dies auch in den Geschäftsordnungen einiger anderer europäischer Parlamente vorgesehen ist ${ }^{10}$. Die am 23. Juli 1970 im Hohen Haus stattgefundene Wahl des Parlamentariers Tassos Papadopoulos zum Stellvertreter des damaligen Präsidenten des Repräsentantenhauses Glafkos Kliridis für jedweden Notfall war allenfalls nur eine vorübergehende (Not-)Lösung; während der Periode Juli bis Dezember 1974 und der Ausübung der staatspräsidialen Amtsgeschäfte durch Kliridis (für den im Exil befindlichen Makarios) übte Papadopoulos die entsprechenden Amtsgeschäfte im Repräsentantenhaus aus ${ }^{11}$ - dies ist bisher eine singuläre Erscheinung der zyprischen Parlamentspraxis geblieben.

\section{Notrechtliche Bewältigung der türkisch-zyprischen Parlamentsabstinenz}

Durch die beschriebene Notlage ab 1963 sahen sich die Republik Zypern und damit auch ihre verfassungsrechtlich vorgesehenen Staatsorgane gezwungen, das Fortbestehen von krisenbetroffenen Teilen der Verfassungsordnung rechtlich zu gewährleisten: Dies geschah schon sehr bald auf der Basis des Notrechts ${ }^{12}$, denn die partielle Unmöglichkeit einer strikt formalrechtlichen Umsetzung der Verfassung hätte sonst den völligen Stillstand des zyprischen Staatsorganisationsrechts zur Folge gehabt. Vor dem Hintergrund der schwierigen Verhältnisse auf der Insel wurde so dem Prinzip „Salus rei publicae suprema lex esto“ des römischen Rechts Genüge getan.

Historische Wurzel der Anwendung dieses Notrechts auf Zypern stellt die Entscheidung des Obersten Gerichtshofes der Insel 1964 im Fall „The Attorney-General of the Republic vs. Mustafa Ibrahim and others" (CLR 194) dar; darin wurde die Verfassungskonformität des Gesetzes Nr. 33/1964 (zur Gewährleistung der Handlungsfähigkeit der Justiz Zyperns), das nach Rücktritt der Vorsitzenden von Verfassungsgerichts- und Oberstem Gerichtshof verabschiedet worden war, geprüft und bejaht ${ }^{13}$. Obwohl Art. 183 der Verfassung - bezüg-

10 Vgl. etwa als relativ aktuelles Beispiel \$1 Abs. 1 der Geschäftsordnung des Hessischen Landtags.

11 Dieser einmalige Zustand muss auch im Kontext der nirgendwo in der Verfassung vorgesehenen Möglichkeit eines Parlamentsvizepräsidenten, den Chef des Abgeordnetenhauses temporär zu vertreten, gesehen werden.

12 Nicht zu verwechseln mit dem - für deutsche Leser - uralten und vielfach belasteten Topos des ius eminens.

13 Infolge des dauerhaften Fernbleibens der türkisch-zyprischen Seite von den Verfassungsorganen der Republik sollte der Oberste Gerichthof auch in den Folgejahren eine reichhaltige Rechtspre- 
lich des Notstands - formal seine Verhängung durch Beschluss des Kabinetts voraussetzt, dies seinerzeit jedoch nicht geschehen war, erkannte der rechtsvergleichend argumentierende Gerichtshof richtigerweise die faktische Existenz einer Notsituation an (insbesondere in Anbetracht der Tatsache, dass die Vereinten Nationen bereits internationale Streitkräfte auf die Insel gesandt hatten, um den Frieden wiederherzustellen) ${ }^{14}$.

Auch das Repräsentantenhaus Zyperns sollte naturgemäß nach einiger Zeit mit der Thematik des Notrechts in Berührung kommen: Es entschied am 20. Juni 1985 nur mit der vorgesehenen Zweidrittelmehrheit der griechisch-zyprischen Parlamentarier per Gesetz Nr. 124/1985 die Erhöhung seiner Mandatszahl auf 8015; infolge der Regelung in Art. 62 Abs. $2^{16}$ stehen nunmehr 56 Sitze griechisch-, 24 türkisch-zyprischen Parlamentariern zu; hinzu kommen die drei beobachtenden Abgeordneten der Armenier, Latiner und Maroniten, von denen noch die Rede sein wird. Da nach der faktischen Teilung der Insel eine für diesen Beschluss erforderliche Zweidrittelmehrheit der türkisch-zyprischen Abgeordneten nicht eingeholt werden konnte, kam die Mandatszahländerung des Hohen Hauses auf der Basis des besagten Notrechts zustande, dessen diesbezügliche Anwendung im europäischen Vergleich einmalig ist. Als Hauptgrund der Erhöhung wurde im damaligen Beschluss plausibel angeführt, dass die Zahl von damals 35 tatsächlich aktiven Abgeordneten zu gering für die effektive parlamentarische Arbeit, insbesondere auch in den verschiedenen Ausschüssen sei $^{17}$.

Ob allerdings in diesem Fall die Anwendung des Notrechts gerechtfertigt war, sollte interessanterweise über Jahre streitig bleiben; erst im Zuge der Entscheidung des Obersten Gerichtshofes im Fall „Nikolaou vs. Nikolaou“ (1992) wurde das Gesetz Nr. 124/1985 offiziell als verfassungskonform anerkannt ${ }^{18}$. Dieser Fall bestätigte im Übrigen auch die Verfassungskonformität anderer strittiger Regelungen, die das Repräsentantenhaus nach 1986 vorgenommen hatte.

chung zur Anwendung des Notrechts produzieren, vgl. etwa den Fall „Ioannis Iossif vs. The Cyprus Telecommunication Authority“ (1970), 3 CLR 225, betreffend des vom Repräsentantenhaus verabschiedeten Gesetzes 33/1967 für den Ausschuss des öffentlichen Dienstes; vgl. zur Thematik des Notrechts unter anderem Andreas Nikola Loizou, Die Verfassung der Republik Zypern (auf Griechisch), Nikosia 2001, S. 12 - 14; Loukis Papaflippou, Notrecht und Verfassungsordnung auf Zypern (auf Griechisch), Nikosia 1995, S. 59 - 65; Achilleus Aimilianidis, a.a.O (Fn. 9), S. $102 \mathrm{ff}$.

14 Bezüglich der vergleichenden Analyse des Notstandsrechts in Deutschland, den USA, England, Frankreich, Italien und Griechenland vgl. auch „The Attorney-General of the Republic vs. Mustafa Ibrahim and others", CLR 194; Achilleus Aimilianidis, a.a.O. (Fn. 9), S. 105 - 107.

15 Vgl. Regierungsblatt der Republik Zypern vom 5. Juli 1985; ebenso unter http://www.parliament.cy/parliamentgr/002_10_07.htm (auf Griechisch) (Abruf am 27. März 2009).

16 Derselbe Abs. 2 sieht als entsprechende Analogie zwischen den Griechen und Türken der Insel einen 70- zu 30-Prozent-Anteil der Parlamentarier zugunsten der griechischen Zyprer vor (wobei die tatsächliche Bevölkerungsanalogie auf Zypern 196078 Prozent griechische, 18 Prozent türkische Zyprer betrug, vgl. unter anderem Iossif Iossif, a.a.O. (Fn. 2), S. 48); diese sind am selben Wahltage getrennt von den beiden betroffenen Bevölkerungsgruppen zu wählen. So kam es in der ersten Parlamentswahl Ende 1960 erstmals (und bisher letztmals) zu der Wahl von 35 griechischund 15 türkisch-zyprischen Abgeordneten, vgl. Dimitrios Parashu, a.a.O. (Fn. 4).

17 Vgl. http://www.parliament.cy/parliamentgr/002_10_07.htm (auf Griechisch) (Abruf am 27. März 2009).

18 Vgl. „Nikolaou vs. Nikolaou“, (1992) 1 AA $\triangle 1338$; Achilleus Aimilianidis, a.a.O (Fn. 9), S. 158; die faktische Aufstockung der Parlamentssitze seit 1985 blieb davon unbeeinflusst. 
Auch hinsichtlich der für Verfassungsänderungen erforderlichen doppelten Zweidrittelmehrheiten im Repräsentantenhaus hat die Praxis Beispiele zu bieten: Wegen der Abwesenheit der türkisch-zyprischen Mandatsträger konnten die drei bis dato stattgefundenen Verfassungsänderungen der Republik nur von den griechisch-zyprischen Abgeordneten beschlossen werden ${ }^{19}$ : 1989 wurde Art. 111 dahingehend geändert, dass nunmehr auch standesamtliche Trauungen vorgenommen werden können; 1996 wurde das aktive Wahlrechtsalter auf 18 Jahre gesenkt (Art. 63) und Art. 66 Abs. 2 verändert, um zeitaufwendige Neuwahlen für einzelne Parlamentsmandate zukünftig zu verhindern. Auch hier war das Notrecht die Basis für die entsprechenden Beschlüsse des Hohen Hauses.

Art. 63 betrifft das für eine Parlamentswahl relevante Wahlvolk: Gemäß Satz 1 konnten alle Zyprer ab dem 21. Lebensjahr ihre Stimme abgeben - nach Änderung von Verfassung und Wahlgesetz ${ }^{20}$ hatten bei der Parlamentswahl von 1996 erstmals alle Zyprer ab dem vollendeten 18. Lebensjahr das aktive Wahlrecht ${ }^{21}$. Die bereits 1985 und 1986 hierzu verabschiedeten Gesetze waren vom Obersten Gerichtshof als nicht verfassungskonform gewertet und die Anwendungsmöglichkeit des Notrechts verneint worden ${ }^{22}$; erst nach dem Fall „Nikolaou“ konnte der Gesetzgeber in Form des neuen Wahlgesetzes von 1996 reagieren ${ }^{23}$.

Die Parlamentswahlprozedur selbst ist in Art. 66 geregelt. In Abs. 1 wird als regulärer Wahltermin stets der zweite Sonntag desjenigen Monats festgesetzt, der unmittelbar vor dem Endmonat der vorherigen Wahlperiode abläuft. Eine im europäischen Vergleich auch eher seltene Lösung bot bis 1996 Abs. $2^{24}$ : Im Falle der Vakanz eines Parlamentssitzes (wie in Art. 71 definiert, also beispielsweise durch Todesfall, schriftlich eingereichten Rücktritt oder nach Annahme etwa eines Regierungs- oder Kommunalamtes) war eine Neuwahl im betreffenden Wahlkreis innerhalb von maximal 45 Tagen nach Auftreten des jeweiligen Vakanzgrundes abzuhalten; das genaue Wahldatum legte das Hohe Haus per Beschluss selbst fest. Als einschlägiges Beispiel kann die Ersatzwahl vom 22. August 1982 im Wahlbezirk Famagusta genannt werden: Nach dem Tod des christdemokratischen Abgeordneten Kostas Hatzikakos wurde Zacharias Toulouras (Dimokratikon Komma, DIKO) neu ins

19 Vgl. http://www.parliament.cy/parliamentgr/002_04.htm (auf Griechisch) (Abruf am 27. März 2009).

20 Per entsprechendem Gesetz Nr. 106 (I)/1996, vgl. http://www.parliament.cy/parliamentgr/002_09.htm\#A063 (auf Griechisch) (Abruf am 28. März 2009); wieder auf der Basis des Notrechts.

21 Trotz der seit 1979 bestehenden Wahlpflicht ist es auf Zypern bis zum heutigen Tage nicht zu strafrechtlichen Sanktionen (in Form von Bußgeldern) für Nichtwähler gekommen.

22 Vgl. „President of the Republic vs. House of Representatives“ (1985), 3 CLR 2224; „President of the Republic vs. House of Representatives" (1986), 3 CLR, insbesondere S. 1443 - 1445 des entsprechenden Beschlusses - es bleibt zu konstatieren, dass die Änderungspläne von 1986 erst mit einer Verspätung von zehn Jahren Wirklichkeit werden konnten; ein Beweis dafür, wie schwer sich Justiz und Legislative Zyperns hinsichtlich der Anwendung des Notrechts taten - insbesondere der Oberste Gerichtshof ging im Laufe der Zeit sehr streng mit dessen Anwendungsmöglichkeiten um. Vgl. darüber hinaus den interessanten Fall „President of the Republic vs. House of Representatives" (1985), 3 CLR 2801, in dem der Oberste Gerichtshof die Anwendung des Notrechts für das „Gesetz über Ministerien“ und deren potentielle Benennung durch das Repräsentantenhaus ablehnte.

23 Vgl. unter anderem Achilleus Aimilianidis, a.a.O (Fn. 9), S. 152 f.

24 Vgl. http://www.parliament.cy/parliamentgr/002_09.htm\#A066 (auf Griechisch) (Abruf am 30. März 2009). 
Parlament gewählt, nachdem sich auch das kommunistische Anorthotikon Komma Ergazomenou Laou (AKEL) für seine Wahl ausgesprochen hatte ${ }^{25}$.

1996 entschloss sich das Parlament zu einer ebenfalls auf dem Notrecht basierenden Änderung von Art. 66. Absatz 2 sieht nunmehr vor, dass ein vakanter Parlamentssitz innerhalb von maximal 45 Tagen nach Auftreten der Vakanz neu zu vergeben ist; das Nähere regelt ein Gesetz ${ }^{26}$. Das neue Wahlgesetz der Republik (Nr. 115 (I)/199627) sieht in diesem Zusammenhang vor, dass ein Nachrücker dem vorherigen Mandatsträger nachfolgen kann. Auch diesbezüglich war ursprünglich schon $1986^{28}$ ein Gesetz vom Repräsentantenhaus verabschiedet worden, das ein Nachrücken für vakante Parlamentssitze vorsah ${ }^{29}$. Nachdem der Oberste Gerichtshof diese Regelung seinerzeit als verfassungswidrig eingestuft hatte ${ }^{30}$, kam es einige Jahre später zu einer Revision auch dieser Rechtsprechung. Der Gerichtshof erklärte im Zuge des bereits bekannten Falls „Nikolaou vs. Nikolaou“ die Nachfolge in das Parlament ohne Neuwahl schließlich für verfassungskonform; nur so war auch für die Legislative der Weg frei, mit dem angesprochenen neuen Wahlgesetz zu reagieren. Im Folgejahr (1997) entschied der Oberste Gerichtshof, dass die Verfassungsänderung selbst gerechtfertigt und verfassungskonform war $^{31}$.

Es bleibt festzustellen, dass in der Praxis eine im ehemaligen Art. 66 Abs. 2 angeführte Neuwahl heutzutage nur noch für die Abgeordneten der Minderheitsgruppen stattfindet, theoretisch wohl auch für die 24 türkisch-zyprischen Sitze des Repräsentantenhauses.

25 Vgl. http://www.parliament.cy/parliamentgr/002_10_06.htm (auf Griechisch) (Abruf am 30. März 2009).

26 Vgl. http://www.parliament.cy/parliamentgr/002_09\#A066 (auf Griechisch) (Abruf am 30. März 2009).

27 Vgl. Konstantinos Goumas / Stelios Koutsiopoulos (Hrsg.), a.a.O. (Fn. 2), hier aber Band 1996 (auf Griechisch), Athen 1996, S. 77; http://www.parliament.cy/parliamentgr/002_10_09.htm (auf Griechisch) (Abruf am 30. März 2009).

28 Vgl. http://www.parliament.cy/parliamentgr/002_01.htm (auf Griechisch) (Abruf am 30. März 2009).

29 Dieses Nachrücken war 1986 und ist heute demjenigen Kandidaten vorbehalten, der nach dem ausscheidenden die meisten Präferenzwählerstimmen auf sich vereinigen konnte.

30 Vgl. hinsichtlich der Verneinung der Möglichkeit einer Anwendung des Notrechts „President of the Republic vs. House of Representatives" (1986), 3 CLR 1439; siehe auch die Entscheidung des Obersten Gerichtshofes Zyperns im Falle „Pavlos Angelidis vs. Christos Petas and others“ (1 CLR, 1988).

31 Vgl. Entscheidung des Obersten Gerichtshofes Zyperns im Falle „Yannakis Koulounti vs. House of Representatives and others" (1997), 1 AA $\triangle 1026$; als jüngstes Beispiel dieser neuen Mandatsnachfolgeregelung seien an dieser Stelle die im März 2008 aufgrund der Berufung in die Regierung Zyperns vom Abgeordnetenmandat zurückgetretenen (Art. 70) Kypros Chryssostomidis, Sotiroula Charalambous, Kostas Papakostas und nicht zuletzt, gemäß Art. 41 Abs. 1, der neu gewählte Staatspräsident Dimitris Christofias selbst genannt, deren Nachfolger im Hohen Haus Stella Misiaouli Dimitriou, Skevi Koutra Koukouma, Yannakis Gavriil sowie der Nachrücker des Wahlkreises Keryneia, Klavdios Mavrochannas, wurden; vgl. dazu den entsprechenden Bericht der Zeitungen Simerini und Phileleftheros vom 7. März 2008 (auf Griechisch); ebenso unter http://www.parliament.cy/parliamentgr/002_10_11.htm (auf Griechisch) (Abruf am 30. März 2009). 


\section{Sonstige parlamentsrechtliche Besonderheiten}

Das Repräsentantenhaus weist im europäischen Vergleich eine ganze Reihe von Besonderheiten auf. Dies trifft nicht nur auf die faktisch nurmehr symbolisch dualistisch angesehenen, sowohl für die griechisch- als auch die türkisch-zyprische Bevölkerungsgruppe getroffenen verfassungsrechtlichen Vorkehrungen zu; insbesondere die präsidiale Staatsform mit der Unabhängigkeit der starken Exekutive vom Parlament ist hier zu nennen. So hat das Repräsentantenhaus beispielsweise nicht die Möglichkeit eines Misstrauensvotums gegenüber der Regierung, wie man es von der überwiegenden Mehrzahl der EU-Mitgliedstaaten gewohnt ist; dem Hohen Haus ist hauptsächlich mittels des alljährlichen Haushaltsgesetzes eine konstante Kontrolle der Exekutive möglich - darüber hinaus mit Anfragen eines Abgeordneten an ein Regierungsmitglied und in der Praxis (im europäischen Vergleich) nicht sehr häufigen Untersuchungsausschüssen.

Als ebenso wichtig ist an dieser Stelle Art. 65 der Verfassung anzusehen, in dem die Legislaturperiode des Hohen Hauses auf fünf Jahre festgesetzt wird. Zur entsprechenden Praxis der Insel ist hier zu erwähnen, dass das Parlament insbesondere nach 1991 regelmäßig die Legislaturperiode voll ausgeschöpft hat ${ }^{32}$ - dies kann im mediterranen, aber auch im Vergleich mit anderen jüngeren EU-Mitgliedern als durchaus beachtlich eingestuft werden. Abs. 2 desselben Artikels betont explizit, dass ein Repräsentantenhaus natürlicherweise bis zur Arbeitsaufnahme des Folgeparlaments im Amt bleibt, aber gemäß Art. 68 nur im dringlichen Notfall legislativ tätig werden darf $\mathrm{f}^{3}$.

In diesem Kontext ist es sinnvoll ${ }^{34}$, auf einen weiteren, für die Wirkungsdauer eines Repräsentantenhauses Zyperns wesentlichen Verfassungsartikel hinzuweisen: Art. $67^{35}$. Das dort angeführte, ebenfalls im europäischen Vergleich seltene Selbstauflösungsrecht des Hohen Hauses (Abs. 1) fordert die absolute Mehrheit der gesetzlichen Mitglieder als Bedingung - worin mindestens ein Drittel der türkisch-zyprischen Abgeordneten vertreten zu sein hat. Da letzteres aufgrund der faktischen Teilung der Insel nicht möglich ist, hat sich bis heute jede vorzeitige Parlamentsauflösung - natürlich basierend auf dem Notrecht - auf die absolute Mehrheit der griechisch-zyprischen Abgeordneten gestützt.

Art. 67 Abs. 2 regelt prozedurale Fragen hinsichtlich der Parlamentswahlfristen nach der vorzeitigen Auflösung: Das neue Repräsentantenhaus muss nach dem entsprechenden Auflösungsbeschluss der benötigten Majorität nicht früher als 30 und nicht später als 40 Tage

32 Vgl. Dimitrios Parashu, a.a.O. (Fn. 4). Abgesehen von der Regelung des Art. 67 Abs. 3.

33 Art. 143 Abs. 1 der Verfassung gibt unter anderem auch dem Repräsentantenhaus selbst (in der Form von mindestens einem Fünftel seiner gesetzlichen Mitglieder, die einen entsprechenden Antrag unterstützen) die Möglichkeit, durch den Obersten Gerichtshof das Vorliegen eines solchen „dringlichen Notfalles“ prüfen zu lassen.

34 Als erstes Beispiel sei hier Folgendes genannt: Die zweite reguläre Parlamentswahl Zyperns sollte erst am 5. Juli 1970 stattfinden - also fünf Jahre nach dem eigentlichen Wahltermin. Dies war mit der per Gesetz vorgesehenen provisorischen Weiterführung der parlamentarischen Geschäfte durch das erste gewählte Parlament nach den folgeschweren Unruhen Mitte der 1960er Jahre zu erklären. Diese Weiterführung der parlamentarischen Geschäfte wurde zwischen 1965 und 1970 alljährlich, insgesamt also fünf Mal, per Gesetz des Hohen Hauses beschlossen. Schon die Staatspräsidentenwahl fand aus demselben Grunde nicht 1965 statt, sondern erst 1968.

35 Dies stellt die einzige verfassungsrechtliche Möglichkeit einer vorzeitigen Parlamentsauflösung auf Zypern dar. 
gewählt werden, das neu gewählte Parlament nicht später als 15 Tage nach dieser Wahl erstmals zusammenzutreten - die Praxis hat in diesem Zusammenhang die stete Einhaltung dieser Fristen mit sich gebracht.

Eine vergleichsweise originelle Vorschrift stellt Abs. 3 des Art. 67 dar: Das nach einer vorzeitigen Auflösung neu gewählte Hohe Haus tritt sein Amt stets nur für die Rest-Legislaturperiode seines Vorgängers an; wenn die Auflösung jedoch im letzten Jahr dieser Periode vorgenommen wurde, ist das neue Repräsentantenhaus sowohl für den Rest als auch für die komplette folgende regelmäßige Legislaturperiode gewählt. Im Fall etwa des $1985^{36}$ gewählten Repräsentantenhauses hatte sich seine Lebensdauer sowohl auf die Periode bis zum September 1986 als auch darüber hinaus bis zum September 1991 zu erstrecken - allerdings sollte dann jedoch bereits im Mai 1991 ein neues Repräsentantenhaus gewählt werden, was einen anderen Hintergrund hat:

Seit 1991 hatte sich die Parlamentspraxis bewährt, Art. 67 Abs. 3 teleologisch differenziert auszulegen: Das Repräsentantenhaus löst sich kurz vor einer Neuwahl selbst auf, hauptsächlich die dann bereits abgearbeitete fünfjährige Legislaturperiode nach Art. 65 beachtend - im April 1991 etwa aufgrund der effektiv längst abgelaufenen fünf Jahre; so auch am 18. April 1996, am 20. April 2001 und am 13. April $2006^{37}$.

Die Historie des Repräsentantenhauses weist, abgesehen von den Ereignissen Mitte der 1960er Jahre ${ }^{38}$, folgende Abweichungen von einer fünfährigen Legislaturperiode auf: Nach 1970 wäre der nächste reguläre Wahltermin $1975^{39}$ gewesen, infolge des Kriegszustands von 1974 konnte dieser jedoch nicht eingehalten werden - ein weiteres Beispiel für eine faktisch länger als fünf Jahre dauernde Legislaturperiode. Als Exempel einer kürzeren parlamentarischen Lebensdauer sind die Neuwahlen des Repräsentantenhauses in den Jahren $1981^{40}$ und $1985^{41}$ zu nennen. Sie waren das Resultat einer erforderlichen, von den vertretenen Fraktionen beschlossenen vorzeitigen Auflösung des Hohen Hauses - 1981 partiell mit der Begründung einer schnellstmöglichen Umsetzung der damals frisch beschlossenen Wahlrechtsreform ${ }^{42}$ - im November $1985^{43}$ nach nur knapp viereinhalbjähriger Legislaturperiode, per einstimmiger Abstimmungsentscheidung, als Reaktion auf den Stand der Gespräche mit den Vertretern des völkerrechtswidrig besetzten Nordens. Bei den Parlamentsauflösungen ab 1991 wurde der für die Fünfjahresdauer relevante Wortlaut des Art. 65 sehr wohl geachtet - im Unterschied zur Auslegung des Art. 67 Abs. 3.

36 Vgl. Dimitrios Parashu, a.a.O. (Fn. 4).

37 Vgl. ebenda. Ebenso etwa unter http://www.parliament.cy/parliamentgr/002_10_08.htm (auf Griechisch) (Abruf am 2. April 2009).

38 Unruhen zwischen Griechen und Türken, die zum effektiven Rückzug der letzteren aus allen verfassungsrechtlich vorgesehenen Organen der Republik führten; vgl. Vangelis Koufoudakis, Das Zypernproblem, die Griechisch-Türkischen Beziehungen und die Großmächte 1960-1986, in: Griechisch-Türkische Beziehungen 1923-1987, Sammelband (auf Griechisch), Athen 1991, S. 221.

39 Vgl. Dimitrios Parashu, a.a.O. (Fn. 4).

40 Vgl. ebenda.

41 Vgl. ebenda.

42 Näheres zu dieser Wahlrechtsreform siehe in Abschnitt 4.

43 Konstantinos Goumas / Stelios Koutsiopoulos (Hrsg.), a.a.O. (Fn. 2), S. 1311, S. 1327; http://www. parliament.cy/parliamentgr/002_01.htm (auf Griechisch) (Abruf am 2. April 2009). 
Für das legislative Wirken ${ }^{44}$ des Hohen Hauses ist Art. 80 Abs. 1 der Verfassung besonders wichtig: Gesetzgebungsinitiativen können sowohl von Abgeordneten (Gesetzesvorschlag) als auch von den Ministern Zyperns (Gesetzentwurf) ausgehen.

Abs. 2 verbietet es den Abgeordneten jedoch, Gesetzesvorschläge bezüglich der Aufstockung der im Staatshaushalt vorgesehenen Ausgaben einzubringen; ebenso kann das Repräsentantenhaus bereits in einem Ergänzungshaushalt vorhandene Kapitel nur annehmen oder ablehnen, ohne einen eigenen Vorschlag einzubringen (Art. 167 Abs. 6); die Auseinandersetzung mit der entsprechenden Gesetzesvorlage eines Finanzministers und ihre notwendige Billigung (Art. 81) bleibt trotzdem einer der wesentlichsten Punkte parlamentarischer Kontrolle auf Zypern. Allerdings zeigt auch hier die Praxis regelmäßig die Übereinstimmung einer großen parlamentarischen Mehrheit mit den jeweiligen Vorlagen auf. Der theoretische Fall, dass ein Staatshaushalt am ersten Tage des betreffenden Jahres immer noch nicht vom Hohen Haus gebilligt und verabschiedet worden ist, würde gemäß Art. 168 Abs. 3 zur Möglichkeit des Repräsentantenhauses führen, das Kabinett per entsprechendem Beschluss innerhalb bestimmter Fristen (maximal zwei Monate) zu gewissen notwendigen Ausgaben zu bevollmächtigen.

Eine zweifelsfrei in diesem Rahmen auch als Besonderheit des Repräsentantenhauses anzuführende Tatsache betrifft die Partizipation von Repräsentanten derjenigen Bevölkerungsminderheiten, die seit den Anfängen der Republik Zypern eine Teilnahme an der griechisch-zyprischen Bevölkerungsgruppe wählten: die orthodoxen Armenier sowie die Latiner (Katholiken, die dem Patriarchat Jerusalems angehören) und Maroniten (ebenfalls Katholiken, die Rom verbunden sind). Im Rahmen des Gesetzes Nr. $58^{45}$ bezüglich der „Repräsentation der religiösen Gruppen“ von 1970 sollen stets jeweils ein Vertreter dieser Gruppen von der jeweiligen Minderheit in das Hohe Haus gewählt werden. Dies war schon in der griechisch-zyprischen Kommunalkammer vorgesehen (Art. 109 der Verfassung von 1960; Art. 3 Abs. 1 des Gesetzes Nr. 8/1960 dieser Kammer ${ }^{46}$ ); seit der angesprochenen Übertragung der Rechte dieses Kommunalparlaments auf das Hohe Haus 1965 (KammerGesetz Nr. 12/1965) ${ }^{47}$ waren die drei Repräsentanten durch alljährliche Verlängerungsbeschlüsse bis 1970 dort ebenfalls vertreten. Per Gesetz Nr. $38^{48}$ des Jahres 1976 ist darüber hinaus ihre regelmäßige zeitliche Mandatsdauer derjenigen des jeweiligen Repräsentantenhauses gleichgestellt, beträgt demnach fünf Jahre ${ }^{49}$; seit 1991 findet ihre Wahl stets zeitgleich mit derjenigen der übrigen Parlamentarier statt - im Unterschied zu letzteren naturgemäß ohne politische Bindung an irgendeine Partei. Die Abgeordneten der Armenier, Latiner und Maroniten nehmen, abgesehen von allen üblichen Rechten und Pflichten eines

44 Der Staatspräsident kann seinerseits die Gesetzgebung des Repräsentantenhauses, insbesondere hinsichtlich der empfindlichen Sektoren der Außen- und Verteidigungspolitik der Insel, per absolutem Vetorecht (Art. 50), in anderen Politikbereichen per aufschiebendem Vetorecht (Art. 51) blockieren.

45 Vgl. Christos Iakovou, Die Institution der religiösen Gruppen in der Verfassung, in: Politis vom 1. Juli 2001 (auf Griechisch).

46 Vgl. Christos Iakovou, a.a.O. (Fn. 45).

47 Vgl. ebenda.

48 Vgl. ebenda.

49 Vgl. den soeben vorgestellten Art. 65 der Verfassung. 
Abgeordneten innerhalb der Mitgliedstaaten der EU ${ }^{50}$, ex officio am Bildungsausschuss des Hohen Hauses teil und äußern sich zu den Problemen und Thematiken, die ihre Bevölkerungsgruppe unmittelbar betreffen, bevor das Repräsentantenhaus diesbezüglich in irgendeine legislative Aktion tritt - sie haben allerdings kein Stimmrecht ${ }^{51}$. Im Hinblick auf ähnliche Minderheitsvertretungen in anderen europäischen Parlamenten (beispielsweise in Polen und Slowenien, auf einem sehr fortgeschrittenen Niveau auch in Schleswig-Holstein) sollte erwogen werden, ob etwa die Einräumung eines Stimmrechts für diese drei Abgeordneten, wenigstens für die Belange der jeweiligen Gruppe, nicht zeit- und sachgemäß wäre. Dies entspräche auch Art. 28 Abs. 1 der Verfassung (Gleichheit aller Bürger vor dem Gesetz) - unabhängig davon, dass es keine offiziell formulierten entsprechenden Forderungen der betroffenen religiösen Gruppen gibt ${ }^{52}$ und der Gleichheitsgrundsatz zweifelsohne in jeder anderen Facette des Lebens der angesprochenen Minderheiten eingehalten wird.

\section{Kurze Geschichte der zyprischen Parlamentswablen seit 1960 und Positionen der Parteien im Prozess der Verhandlungen zur Teilungsüberwindung}

Aufgrund der präsidialen Staatsform der Republik Zypern sind es nicht die Parlamentswahlen, die für eine Regierungsbildung entscheidend sind, sondern die stark personenbezogenen (und zu einem etwas geringeren Maße parteipolitisch gefärbten) Wahlen des Staatspräsidenten. Er ist es, der die Regierung bildet; oftmals ist jedoch die Stärke der Parteien im Hohen Haus ein wertvoller Indikator dafür, welche Mehrheiten in den zumeist nach den Parlamentswahlen stattfindenden Präsidentschaftswahlen gefunden werden können. Diese Mehrheiten kristallisieren sich in der Regel bei der Wahl des Präsidenten des Repräsentantenhauses heraus.

Der aktuelle Staatspräsident Dimitris Christofias führt beispielsweise eine Koalition seines kommunistischen AKEL, des zentristischen DIKO sowie des sozialdemokratischen EDEK (KI.SOS.) an - diese Parteien hatten in der letzten Parlamentswahl 2006 insgesamt 57,9 Prozent der Stimmen sowie 34 (von 56 besetzten) Sitzen im Repräsentantenhaus errungen $^{53}$. Die Parteien, die speziell seit 1976 die parlamentarische und politische Landschaft Zyperns dominieren, sind nach Rangfolge ihres Alters ${ }^{54}$

- die kommunistische Partei AKEL (Anorthotikon Komma Ergazomenou Laou / Wiederaufrichtende Partei des Arbeitenden Volkes), 1926 als Kommunistische Partei Zyperns gegründet und 1931 von der britischen Kolonialmacht für verboten erklärt dienstältester Generalsekretär der Partei war zwischen 1949 und 1988 Ezekias Papaioannou;

50 So etwa Immunität und Indemnität, vgl. Art. 83; angemessene Vergütung, vgl. Art. 84; vgl. http://www.parliament.cy/parliamentgr/002_10_02.htm (auf Griechisch) (Abruf am 10. April 2009).

51 Vgl. http://www.parliament.cy/parliamentgr/002_10_02.htm (auf Griechisch) (Abruf am 11. April 2009).

52 Vgl. unter anderem http://www.armenianmp.com/index.htm?p=49 (Abruf am 11. April 2009); Interview von Benito Mantovani, in: The Cyprus Weekly vom 1. September 2000.

53 Vgl. Dimitrios Parashu, a.a.O. (Fn. 4).

54 Vgl. ebenda. 
- die kleine, erst sozialistische, dann sozialdemokratische EDEK (Eniea Dimokratiki Enosis Kentrou / Vereinigte Demokratische Zentrumsunion), 1970 von Vassos Lyssaridis gegründet, 2001 als KISOS (Kinima Sosialdimokraton) bei den Parlamentswahlen angetreten;

- der christdemokratische DISY (Dimokratikos Synagermos / Demokratische Sammlung), 1976 von Glafkos Kliridis, Präsident des Repräsentantenhauses mit der historisch längsten Amtszeit, gegründet, der bis zu Beginn seiner Staatspräsidentschaft 1993 den Vorsitz der Partei innehatte;

- das zentristische DIKO (Dimokratikon Komma / Demokratische Partei), ebenfalls 1976 von Spyros Kyprianou gegründet und in den letzten Jahren zumeist „Zünglein an der Waage" hinsichtlich der Bildung einer Mitte-Rechts oder Mitte-Links-Regierung darüber hinaus die Partei, die bis heute die relativ meisten Staatspräsidenten stellte.

\begin{tabular}{|c|c|c|c|c|c|}
\hline Jahr & AKEL & DISY & DIKO & EDEK (KISOS) & Andere \\
\hline \multirow[t]{2}{*}{1960} & 35,0 & 56,1 (als PM) & & & 8,9 \\
\hline & 5 Sitze & 30 Sitze & & & - \\
\hline \multirow[t]{2}{*}{1970} & 39,8 & 25,5 (als Eniaion) & 17,9 (als PP) & 13,4 & 3,4 \\
\hline & 9 Sitze & 15 Sitze & 7 Sitze & 2 Sitze & 2 Sitze \\
\hline \multirow[t]{2}{*}{1976} & $\begin{array}{l}\text { 69,5 (mit DIKO } \\
\text { und EDEK) }\end{array}$ & 27,6 (mit DEK) & & & \\
\hline & 34 Sitze & - & & & 1 Sitz \\
\hline \multirow[t]{2}{*}{1981} & 32,8 & 31,9 & 19,5 & 8,2 & 7,6 \\
\hline & 12 Sitze & 12 Sitze & 8 Sitze & 3 Sitze & - \\
\hline \multirow[t]{2}{*}{1985} & 27,4 & 33,6 & 27,7 & 11,1 & 0,2 \\
\hline & 15 Sitze & 19 Sitze & 16 Sitze & 6 Sitze & - \\
\hline \multirow[t]{2}{*}{1991} & 30,6 & 35,8 & 19,6 & 10,9 & 3,1 \\
\hline & 18 Sitze & 20 Sitze & 11 Sitze & 7 Sitze & - \\
\hline \multirow[t]{2}{*}{1996} & 33,0 & 34,5 & 16,4 & 8,1 & 8,0 \\
\hline & 19 Sitze & 20 Sitze & 10 Sitze & 5 Sitze & 2 Sitze \\
\hline \multirow[t]{2}{*}{2001} & 34,7 & 34,0 & 14,8 & 6,5 (KISOS) & 10,0 \\
\hline & 20 Sitze & 19 Sitze & 9 Sitze & 4 Sitze & 4 Sitze \\
\hline \multirow[t]{2}{*}{2006} & 31,1 & 30,3 & 17,9 & 8,9 & 11,8 \\
\hline & 18 Sitze & 18 Sitze & 11 Sitze & 5 Sitze & 4 Sitze \\
\hline \multicolumn{6}{|c|}{$\begin{array}{l}\text { Anmerkung: AKEL = Anorthotikon Komma Ergazomenou Laou / Wiederaufrichtende Partei des Arbei- } \\
\text { tenden Volkes (Kommunisten), DISY = Dimokratikos Synagermos / Demokratische Sammlung (Christ- } \\
\text { demokraten), DIKO = Dimokratikon Komma / Demokratische Partei (Zentristen), EDEK (KISOS) = } \\
\text { Eniea Dimokratiki Enosis Kentrou (Kinima Sosialdimokraton) / Vereinigte Demokratische Zentrums- } \\
\text { union (Bewegung der Sozialdemokraten / Sozialdemokraten). Die Parteien werden nach ihrer Reihenfol- } \\
\text { ge in der letzten Wahl zum Repräsentantenhaus angeführt. } \\
\text { Quelle: Dimitrios Parashu, a.a.O. (Fn. 4). }\end{array}$} \\
\hline
\end{tabular}




\begin{tabular}{|c|c|}
\hline Staatspräsident & Regierung (bzw. Regierungskoalition) \\
\hline 1959(1960)-1965/68 Makarios III. & $\begin{array}{l}\text { 1959(1960)-1965/68 Überparteiliche Regierung* } \\
\text { mit Unterstützung von AKEL und PM }\end{array}$ \\
\hline 1968-1973 Makarios III. & $\begin{array}{l}\text { 1968-1973 Überparteiliche Regierung mit Unterstüt- } \\
\text { zung von AKEL, PM / Eniaion, PP und EDEK }\end{array}$ \\
\hline 1973-1977 Makarios III. & $\begin{array}{l}\text { 1973-1976 Überparteiliche Regierung mit Unter- } \\
\text { stützung von AKEL, Eniaion, PP und EDEK } \\
\text { 1976-1977 Überparteiliche Regierung mit Unter- } \\
\text { stützung von AKEL, PP / DIKO und EDEK }\end{array}$ \\
\hline 1977-1978 Spyros Kyprianou (DIKO) & $\begin{array}{l}\text { 1977-1978 Überparteiliche Regierung mit Unter- } \\
\text { stützung von AKEL, DIKO, EDEK }\end{array}$ \\
\hline 1978-1983 Spyros Kyprianou (DIKO) & $\begin{array}{l}\text { 1978-1980 Überparteiliche Regierung mit Unter- } \\
\text { stützung von AKEL, DIKO, EDEK } \\
1980 \text { Überparteiliche Regierung mit Unterstützung } \\
\text { von DIKO und EDEK } \\
\mathbf{1 9 8 0 - 1 9 8 3 ~ A K E L ~ u n d ~ D I K O * * ~}\end{array}$ \\
\hline 1983-1988 Spyros Kyprianou (DIKO) & $\begin{array}{l}\text { 1983-1984 AKEL und DIKO } \\
\text { 1984-1988 DIKO }\end{array}$ \\
\hline 1988-1993 Georgios Vassiliou (unabhängig) & $\begin{array}{l}\text { 1988-1993 Regierung hauptsächlich von } \\
\text { Unabhängigen, unterstützt von AKEL und EDEK }\end{array}$ \\
\hline 1993-1998 Glafkos Kliridis (DISY) & $\begin{array}{l}\text { 1993-1997 DISY und DIKO } \\
\text { 1997-1998 DISY }\end{array}$ \\
\hline 1998-2003 Glafkos Kliridis (DISY) & $\begin{array}{l}\text { 1998-1999 DISY (+KF), DIKO, EDEK, EDI } \\
\text { 1999-2003 DISY, DIKO, EDI }\end{array}$ \\
\hline 2003-2008 Tassos Papadopoulos (DIKO) & $\begin{array}{l}\text { 2003-2007 AKEL, DIKO, EDEK } \\
\text { 2007-2008 DIKO, EDEK }\end{array}$ \\
\hline seit 2008 Dimitris Christofias (AKEL) & seit 2008 AKEL, DIKO, EDEK \\
\hline \multicolumn{2}{|c|}{$\begin{array}{l}\text { * „Uberparteilich“, da der zyprische Staatspräsident ohnehin bei der Wahl seiner Minister nicht gebun- } \\
\text { den ist - erst in den letzten beiden Jahrzehnten wurden Regierungskoalitionen gebildet. } \\
\text { ** Auf der Basis des „Minimum-Programms“ zum Zweck der Wiederwahl Kyprianous } 1983 \text {. } \\
\text { Anmerkung: AKEL = Anorthotikon Komma Ergazomenou Laou / Wiederaufrichtende Partei des Arbei- } \\
\text { tenden Volkes (Kommunisten), DISY = Dimokratikos Synagermos / Demokratische Sammlung (Christ- } \\
\text { demokraten), DIKO = Dimokratikon Komma / Demokratische Partei (Zentristen), EDEK (KISOS) = } \\
\text { Eniea Dimokratiki Enosis Kentrou (Kinima Sosialdimokraton) / Vereinigte Demokratische Zentrums- } \\
\text { union (Bewegung der Sozialdemokraten / Sozialdemokraten), PM = Patriotiko Metopo / Patriotische } \\
\text { Front (liberal-konservativ), Eniaion = Einheitliche Partei (liberal-konservativ), PP = Proodeftiki Parataxis } \\
\text { / Fortschrittliche Partei (liberal-zentristisch), DEK = Dimokratikon Ethnikon Komma / Demokratische } \\
\text { Nationale Partei (Christdemokraten), KF = Komma Fileleftheron (Partei der Liberalen, seit } 1998 \text { Teil } \\
\text { von DISY), EDI=Enomeni Dimokrates / Vereinigte Demokraten (liberal, mitentstanden aus dem Kini- } \\
\text { ma Eleftheron Dimokraton / Bewegung Freier Demokraten). } \\
\text { Quelle: Dimitrios Parashu, a.a.O. (Fn. 4). }\end{array}$} \\
\hline
\end{tabular}


Die erste Parlamentswahl der neuen Republik fand am 31. Juli 196055 statt; in den sechs Wahlbezirken (Nikosia, Famagusta, Limassol, Larnaka, Pafos und Kyrenia ${ }^{56}$ ) wurden seinerzeit insgesamt 50 Abgeordnete gewählt - 35 auf griechischer sowie 15 auf türkischer Seite. Durch die damals im Wahlgesetz vorgeschriebene (und wohl durch das britische wie auch von Elementen des zeitgenössischen griechischen und türkischen Wahlsystems beeinflusste) Mehrheitswahl gelang es der liberal-konservativen Patriotischen Front (Patriotiko Metopo), das Gros der den griechischen Zyprern zustehenden Sitze zu erringen; die 15 Abgeordneten der türkischen Zyprer wurden ohne eine entsprechende parteipolitische Unterstützung in das Hohe Haus gewählt, allein ein Drittel von ihnen kam aus der Hauptstadt Nikosia. Am darauf folgenden 16. August fand die konstituierende Sitzung des Repräsentantenhauses statt ${ }^{57}$.

Die nächste Parlamentswahl sollte aufgrund bereits genannter Umstände - und den daraus resultierenden, ab 1965 jährlichen Verlängerungsbeschlüssen der Legislaturperiode durch das Hohe Haus - erst 1970 stattfinden. Dabei gewann die Nachfolgepartei des Patriotiko Metopo, das liberal-konservative Bündnis Eniaion unter Parlamentspräsident Kliridis, begünstigt durch das damalige umständliche Sitzverteilungssystem die relative Mehrheit der griechisch-zyprischen Sitze. Die liberale Fortschrittliche Partei (Proodeftiki Parataxis), ein Teilvorläufer von DIKO, schaffte bei ihrer ersten Wahlteilnahme einen Achtungserfolg. Wahlen für die 15 Mandate der türkischen Zyprer fanden nicht statt; ihre Sitze blieben in der Folge weiterhin (bis zum heutigen Tag) unbesetzt ${ }^{58}$. Nach den dramatischen Geschehnissen des Sommers 1974 und der faktischen geographischen Teilung der Insel kam es erst am 5. September $1976^{59}$ zur dritten Parlamentswahl der Republik (kurze Zeit zuvor war Parlamentspräsident Kliridis zurückgetreten und Tassos Papadopoulos vom Haus

55 Vgl. ebenda.

56 Die Wahlbezirke Zyperns haben sich trotz der faktischen Teilung seit 1974 numerisch und namentlich nicht verändert: Nikosia entsendet 21 (bis 1985 zwölf) griechisch-zyprische Abgeordnete in das Repräsentantenhaus, Limassol zwölf (vormals sieben), Famagusta elf (vormals sieben), Larnaka fünf (vormals vier), Pafos vier (vormals drei) und Kyrenia deren drei (vormals zwei); vgl. unter anderem http://www.parliament.cy/ parliamentgr/002_08_02.htm (auf Griechisch) (Abruf am 16. April 2009).

57 Präsident dieser Sitzung war der älteste Abgeordnete auf griechischer Seite, Charidimos Hatzicharos - sein Vizepräsident wurde der älteste Parlamentarier der türkischen Zyprer, Niazi Maniera (vgl. Art. 72 Abs. 3 der Verfassung hinsichtlich der temporären Vakanz des Präsidiums, die von den jeweils ältesten Parlamentariern von Griechen und Türken Zyperns zu füllen ist). Am selben Tage wurde darüber hinaus von den griechisch-zyprischen Abgeordneten mehrheitlich beschlossen, dass der Konservative Glafkos Kliridis für diese erste Legislaturperiode das Amt des Parlamentspräsidenten übernehmen würde, als sein Vizepräsident wurde auf türkischer Seite Orhan Muderisoglou gewählt; vgl. unter anderem http://www.parliament.cy/parliamentgr/003_01_02.htm (auf Griechisch) (Abruf am 18. April 2009). Staatspräsident war, schon seit der entsprechenden Wahl vom 13. Dezember 1959, Erzbischof Makarios III.; er wurde in der Folge für zwei weitere Amtszeiten bestätigt.

58 Vgl. Dimitrios Parashu, a.a.O. (Fn. 4). Als Präsident des Hohen Hauses wurde Glafkos Kliridis bestätigt, vgl. http://www.parliament.cy/parliamentgr/003_01_02.htm (auf Griechisch) (Abruf am 20. April 2009); ein (gemäß der Verfassung) türkisch-zyprischer Vizepräsident der Kammer konnte naturgemäß nicht gewählt werden. In der Legislaturperiode zwischen 1970 und 1976 waren zudem Ioannis Mavridis (Maroniten), Andranik Astzian (Armenier) und Anthony Pietroni (Latiner) die Repräsentanten der Minderheiten im Hohen Hause, vgl. http://www.parliament.cy/ parliamentgr/002_10_02.htm (auf Griechisch) (Abruf am 20. April 2009).

59 Vgl. Dimitrios Parashu, a.a.O. (Fn. 4). 
einstimmig zu seinem Nachfolger für die restliche Legislaturperiode gewählt worden ${ }^{60}$ ); die 35 Sitze der griechisch-zyprischen Seite gingen allesamt an den Mitte-Links-Block der Parteien DIKO, AKEL und EDEK sowie Tassos Papadopoulos als unabhängigem Parlamentarier Nikosias (2003 wurde er zum Staatspräsidenten Zyperns gewählt).

Der Block der Christdemokraten (DISY) und DEK (Dimokratikon Ethnikon Komma / Demokratische Nationale Partei) ging infolge des damals nach wie vor bestehenden Mehrheitswahlrechts komplett leer aus - ein Denkzettel für die nach Ansicht vieler Beobachter zu moderate Haltung des ehemaligen Parlamentspräsidenten und DISY-Gründers Kliridis im Rahmen der Gespräche hinsichtlich der Zukunft Zyperns ${ }^{61}$. Der aus dem Exil zurückgekehrte Makarios stützte sich in der Folge maßgeblich auf AKEL, DIKO und EDEK wobei auch DISY dem Abkommen von 1977 betreffend des Verhandlungsziels einer bikommunalen, einheitlichen Föderation auf Zypern nicht ablehnend gegenüberstand.

In dieser Legislaturperiode wurden während der Jahre 1979 bis 1981 mit einer Reihe von Gesetzen des Hohen Hauses (Gesetze Nr. 72/1979, 73/1980 und 16/1981 ${ }^{62}$ ) sowohl die Wahlpflicht für die zyprische Bevölkerung als auch ein verstärktes Verhältniswahlrecht eingeführt - und damit wurde das ursprüngliche Mehrheitswahlrecht ad acta gelegt. Diese Neuerungen kamen bei der nächsten Parlamentswahl nach der vorzeitigen Auflösung des Hauses gemäß Art. 67 Abs. 1 der Verfassung am 24. Mai 1981 zur Anwendung (hauptsächlich infolge der angesprochenen wahlrechtlichen Neuerungen, aufgrund derer die politischen Kräfte rasch einen neuen Wählerauftrag erlangen wollten). Resultat war die Sitzparität zwischen AKEL und DISY, denn beide Parteien hatten bei den Stimmenanteilen kräftig zugelegt; die noch relativ junge Regierungszusammenarbeit zwischen Staatspräsident Kyprianous DIKO und AKEL (im Grunde ein Bündnis des „kleinsten gemeinsamen Nenners“, um die Wiederwahl des Präsidenten 1983 zu sichern) wurde fortgesetzt ${ }^{63}$. Einmütig

60 Vgl. http://www.parliament.cy/parliamentgr/003_01_02.htm (auf Griechisch) (Abruf am 22. April 2009).

61 Als neuer Präsident des Repräsentantenhauses wurde Spyros Kyprianou (von der DIKO) einstimmig gewählt, vgl. http://www.parliament.cy/parliamentgr/003_01_02.htm (auf Griechisch) (Abruf am 22. April 2009); nach dem Tod von Staatspräsident Makarios III. wurde Kyprianou 1977 der zweite Staatspräsident Zyperns (für die restliche Amtsperiode seines verstorbenen Vorgängers) und 1978 in diesem Amt bestätigt, vgl. Dimitrios Parashu, a.a.O. (Fn. 4). Er durfte somit gemäß Art. 41 Abs. 1 der Verfassung nicht mehr dem Repräsentantenhaus angehören; sein Nachfolger als Vorstand des Hohen Hauses wurde Alekos Michailidis (damals DIKO) - zunächst provisorisch, seit dem 22. September 1977 für die gesamte restliche Legislaturperiode; vgl. http://www.parliament.cy/parliamentgr/003_01_02.htm (auf Griechisch) (Abruf am 22. April 2009). Die Abgeordneten der Maroniten und Armenier wurden in ihrem Amt bestätigt; neuer Repräsentant der Latiner wurde Felix Kyrillis; vgl. http://www.parliament.cy/parliamentgr/002_10_03.htm (auf Griechisch) (Abruf am 22. April 2009).

62 Vgl. http://www.parliament.cy/parliamentGR/005_03_02_02/005_03_02_02_097.htm (auf Griechisch) (Abruf am 23. April 2009).

63 Die Wahl des Abgeordneten der Armenier konnte nicht, wie zunächst geplant, zusammen mit denjenigen der Latiner und Maroniten am 27. September 1981 abgehalten werden, wurde jedoch am 14. März 1982 nachgeholt: Aram Kalaitzian wurde als Nachfolger von Andranik Astzian von den Armeniern Zyperns in sein Amt gewählt. Die Maroniten entsendeten erstmals Ninos Hatziroussos, die Latiner erneut Felix Kyrillis in das Hohe Haus; vgl. http://www.parliament.cy/ parliamentgr/002_10_05.htm (auf Griechisch) (Abruf am 23. April 2009); für die neue Legislaturperiode wurde Georgios Ladas (DIKO) Präsident des Repräsentantenhauses; vgl. http://www. parliament.cy/parliamentgr/003_01_02.htm (auf Griechisch) (Abruf am 24. April 2009). 
bewirkten die beiden Partner die Verabschiedung der UN-Resolution 37/253. 1984 wurde dieses Bündnis von Kyprianou aufgrund von zwischenparteilichen Differenzen hinsichtlich der Zyperngespräche jedoch aufgekündigt. AKEL hielt den Vorschlag von UN-Generalsekretär Perez de Cuellar, eine föderale Republik zweier Zonen zu schaffen, für akzeptabel, und auch die oppositionellen Christdemokraten sprachen sich dafür aus; Kyprianou selbst war, nach anfänglich positiver Rezeption des Plans, in der Folge allerdings mit einigen Teilpunkten nicht einverstanden, etwa dem vorgesehenen lediglichen Teilabzug des türkischen Militärs von der Insel. Die Haltung des Präsidenten wurde, abgesehen von der Opposition, auch in den eigenen Reihen kritisiert. Nach Ende des Parteienbündnisses stützte er sich im Rahmen seines Regierungskabinetts nurmehr auf Politiker von DIKO; dies blieb natürlich nicht ohne Folgen.

Die Legislaturperiode sollte anstatt 1986 schon Ende 1985 vorzeitig beendet werden ${ }^{64}$. Den Fraktionen der AKEL und DISY gelang es, auch die übrigen Abgeordneten des Hohen Hauses von der Notwendigkeit einer vorgezogenen Parlamentsauflösung zu überzeugen; so kam es am 1. November 1985 wiederum zu einem solchen einstimmigen Parlamentsbeschluss, und Neuwahlen wurden für den 8. Dezember $1985^{65}$ anberaumt. Diese Entwicklung kann als Anfang vom Ende der Regierung von Staatspräsident Kyprianou angesehen werden. AKEL unterstützte ihn bei der nächsten Präsidentschaftswahl (1988) ${ }^{66}$ nicht mehr, sondern sprach stattdessen dem Unabhängigen Georgios Vassiliou das Vertrauen aus.

Die Parlamentswahl von 1985 wurde wiederum mit dem Wahlsystem des verstärkten Verhältniswahlrechts abgehalten. Interessanterweise stellte sich die Bevölkerung zu einem respektablen Teil hinter die Partei DIKO von Präsident Kyprianou und strafte insbesondere AKEL mit einem Minus von 5,4 Prozentpunkten ab. Wichtigster Grund dürfte die an der Glaubwürdigkeit der Partei kratzende Zusammenarbeit der Kommunisten mit dem, einstmals als „faschistisch“ verschrieenen, politischen Gegenpol DISY anlässlich der Parlamentsauflösungsinitiative gewesen sein. Erstmals konnten sich die Zentristen Kyprianous somit den zweiten Platz in der Wählergunst sichern ${ }^{67}$.

Infolge der Wahl Vassilious zum Staatspräsidenten, der als Akt des guten Willens gegenüber den Zentristen den unabhängigen Außenminister George Iakovou im Amt behielt, kam erneut Bewegung in die Zyperngespräche, die allerdings wiederum nicht von Erfolg gekrönt sein sollten. Im Wesentlichen waren die Parteien (abgesehen von der partiellen Skepsis von AKEL in Europafragen) mit der Linie des Staatsoberhaupts einig, insbesondere in der Auffassung, dass der 1990 erfolgte Antrag Zyperns auf Aufnahme in die Europäischen Gemeinschaften ein wichtiger Schritt zur Lösung der Zypernfrage sei.

64 Vgl. Dimitrios Parashu, a.a.O. (Fn. 4).

65 Ebenda.

66 Ebenda.

67 Vgl. ebenda. Wie gesehen, wurde die Zahl der Abgeordneten gemäß Gesetz Nr. 124/1985 ab dieser Wahl signifikant erhöht; zum Präsidenten des Repräsentantenhauses wurde Vassos Lyssaridis von der sozialdemokratischen EDEK gewählt - aufgrund seiner insgesamt drei Kontrahenten um das Amt war erstmals ein dritter Wahlgang nötig. Gemäß vorherigem einstimmigen Beschluss des Hohen Hauses reichte ihm dort die relative Mehrheit von 22 Stimmen; vgl. http://www.parliament.cy/parliamentgr/003_01_02.htm (auf Griechisch) (Abruf am 2. Mai 2009). Die seit 1981 (respektive 1982) im Amt befindlichen Mandatsträger der Minderheiten wurden allesamt bestätigt; vgl. http://www.parliament.cy/parliamentgr/002_10_07.htm (auf Griechisch) (Abruf am 2. Mai 2009). 
Aufgrund von Art. 67 Abs. 3 der Verfassung hätte eine reguläre Parlamentswahl erst im September 1991 angestanden. Das Hohe Haus löste sich jedoch infolge der effektiv bereits abgearbeiteten fünf Jahre der Legislaturperiode auf, und die Wahl fand am 19. Mai 1991 statt $^{68}$. Wiederum wurde nach dem verstärkten Verhältniswahlsystem gewählt, und erneut gelang es nur vier Parteien, Abgeordnete ins Repräsentantenhaus zu entsenden. Als relativer Gewinner gingen wiederum die Christdemokraten (DISY) aus der Wahl hervor; und trotz des prozentualen Absturzes von DIKO kristallisierte sich heraus, dass nach der nächsten Präsidentschaftswahl eine Koalitionsregierung aus DISY und DIKO entstehen würde ${ }^{69}$. Die folgende Wahl von Glafkos Kliridis zum Staatsoberhaupt im Februar 1993 brachte es zudem mit sich, dass am 4. März 1993 eine ganze Reihe von DISY-Abgeordneten gemäß Art. 70 und 71d der Verfassung auf ihr Mandat verzichteten, um in die neue Mitte-RechtsRegierung des Inselstaats eintreten zu können ${ }^{70}$.

Am 26. Mai $1996^{71} \mathrm{kam}$ es zur nächsten Neuwahl (der Wahltermin des Septembers wurde erneut aufgrund der bereits nahezu komplett abgearbeiteten fünf Jahre vier Monate vorgezogen, und das Repräsentantenhaus löste sich im April einstimmig auf). Das Wahlsystem wurde per Gesetz Nr. 11 (I)/1996 diesmal dahingehend geändert, dass seither von einem beinahe klassischen Verhältniswahlsystem auf Zypern die Rede sein kann, in dem die Wahlberechtigten bis zu drei Präferenzstimmen für Kandidaten einer jeweiligen Parteiliste abgeben können ${ }^{72}$. DISY und DIKO konnten ihre Mehrheit halten; AKEL schaffte es mit 19 Mandaten, den 1991 zurückeroberten zweiten Platz in der Wählergunst zu festigen; EDEK musste einen leichten Rückgang auf fünf Parlamentssitze hinnehmen, und erstmals gelang es einer fünften Partei, den liberalen Vereinigten Demokraten EDI (Enomeni Dimokrates), zwei Sitze zu erlangen ${ }^{73}$.

Die Zypern-Verhandlungen waren derweil auf einem Tiefpunkt der Passivität angekommen; auch die Einladung Kliridis' an einige der kleineren Parteien, auch außerhalb des Mitte-Rechts-Spektrums, an der Regierung teilzunehmen, hatte keine neuen Impulse zur Folge. Vielmehr verließ EDEK bereits 1999 die Koalition, da sie unter anderem mit der

68 Vgl. Dimitrios Parashu, a.a.O. (Fn. 4).

69 Vgl. ebenda.

70 Vgl. http://www.parliament.cy/parliamentgr/002_10_08.htm (auf Griechisch) (Abruf am 2. Mai 2009); vgl. dort ebenso hinsichtlich folgender Fakten: Nach dem Tod des wiedergewählten Abgeordneten der Minderheit der Armenier Aram Kalaitzian am 10. September 1995 kam es am 22. Oktober zu einer Neuwahl für diesen Parlamentssitz, den Petros Kalaitzian erringen konnte. Ioannis Pogiatzis (Maroniten) und Benito Mantovani (Latiner) waren in dieser Periode die beiden anderen Mandatsträger dieser Minderheiten. Zum Präsidenten des Hohen Hauses bis 1996 wurde Alexis Galanos (DIKO) gewählt - er vereinte naturgemäß die Stimmen des Mitte-Rechts-Lagers auf sich.

71 Vgl. Dimitrios Parashu, a.a.O. (Fn. 4).

72 Vgl. ebenda.

73 Vgl. ebenda. Armenier und Latiner bestätigten ihre jeweiligen Abgeordneten; Antonios Hatziroussos wurde von den Maroniten ins Parlament gewählt; vgl. http://www.parliament.cy/parliamentgr/002_10_09.htm (auf Griechisch) (Abruf am 5. Mai 2009). Spyros Kyprianou, der Alt-Staatspräsident und ehemalige Präsident des Repräsentantenhauses, wurde erneut Vorsitzender des Hohen Hauses - ein Amt, das er krankheitsbedingt 2000 niederlegen musste; Dimitris Christofias (AKEL) wurde sein Nachfolger, vgl. http://www.parliament.cy/parliamentgr/003_01_02.htm (auf Griechisch) (Abruf am 6. Mai 2009), womit sich seinerseits schon andeutete, dass nach der nächsten Staatspräsidentschaftswahl im Februar 2003 eine Mitte-Links-Mehrheit Zypern regieren würde. 
Verhandlungsführung von Kliridis nicht einverstanden war - in der Sache war man sich allerdings durchaus einig: Eine bikommunale Föderation war nach wie vor von allen politischen Kräften das erklärte Ziel.

Am 27. Mai $2001^{74}$ folgte die nächste Parlamentswahl der Inselrepublik. Es gelang dabei AKEL, Platz 1 in der Wählergunst zu erringen; DISY und DIKO hatten leichte Verluste hinzunehmen, ebenso KISOS (EDEK) (Kinima Sosialdimokraton; vier Sitze) und die EDI (nurmehr ein Sitz), was wohl an der zwischenzeitlichen Regierungsbeteiligung dieser kleineren Parteien in der „größeren“ Koalition mit DISY und DIKO unter Staatspräsident Kliridis ab 1998 gelegen haben dürfte. Erstmals im Repräsentantenhaus vertreten waren nun die liberale Partei der Mitte NEO (Neoi Orizontes / Neue Horizonte; ein Sitz), die kleine konservative Partei ADIK (Agonistiko Dimokratiko Kinima / Kämpferische Demokratische Bewegung, ein Mandat) sowie die grüne Partei der Bewegung der Ökologen (KOP / Kinima Oikologon Perivallontiston) mit ebenfalls einem Sitz ${ }^{75}$.

Am 21. Mai $2006^{76}$ fand die bisher letzte Wahl zur Vouli ton Antiprosopon / Temsilciler Meclisi statt - wiederum nach dem Ausfüllen der fünfährigen Legislaturperiode. Auch hier war das Verhältniswahlsystem ausschlaggebend: AKEL konnte den ersten Platz verteidigen, trotz eines satten Minus von 3,6 Punkten und zwei verlorenen Parlamentssitzen - die Regierungsbeteiligung seit 2003 hatte teilweise ihren Tribut gefordert. Koalitionspartner DIKO konnte stattdessen um 3,1 Punkte und zwei Sitze zulegen, vor allem wegen des Rückhalts, über den der damalige Staatspräsident und DIKO-Vorsitzende Tassos Papadopoulos in der Bevölkerung verfügte. Seine ablehnende Haltung in Hinblick auf den Plan Kofi Annans zur Wiedervereinigung der Insel im Stil einer Konföderation wurde von vielen als richtig empfunden, stieß allerdings auf die Kritik der anderen großen (und kleineren) Parteien Zyperns (die sich insofern inkonsequent zum bisherigen Ziel der Föderation verhielten). Auch der sozialdemokratische Koalitionspartner EDEK (KISOS) konnte auf niedrigem Niveau um 2,4 Prozentpunkte und einen Sitz zulegen. Die Christdemokraten des DISY verloren trotz ihrer Oppositionsrolle 3,7 Punkte und einen Sitz, verfügen also seither wie AKEL über 18 Mandate $^{77}$ - die unterstützende Haltung von Oppositionsführer Nikos Anastasiadis zum Annan-Plan wurde von den Wählern offenbar nicht im erwünschten Maß gutgeheißen. Die liberale, in Europäische Partei (EVROKO) umbenannte NEO konnte ihre Sitzzahl verdreifachen; die Partei der Ökologen vermochte ihr Wahlergebnis von 2001 zu halten und entsendet nach wie vor einen Abgeordneten in das Hohe Haus ${ }^{78}$.

74 Vgl. Dimitrios Parashu, a.a.O. (Fn. 4).

75 Vgl. ebenda. Zum Abgeordneten der armenischen Minderheit wurde Petros Kalaitzian wiedergewählt; nach seinem Tod am 1. September 2005 wurde im Rahmen der vorgeschriebenen Nachwahl durch die Minderheit der Armenier Zyperns Vahak Atamian am 9. Oktober 2005 zu seinem Nachfolger bestimmt. Abgeordneter der maronitischen Minderheit wurde erneut Antonios Hatziroussos; die römisch-katholischen Latiner wählten wiederum Benito Mantovani; vgl. http:// www.parliament.cy/ parliamentgr/002_10_10.htm (auf Griechisch) (Abruf am 10. Mai 2009). Dimitris Christofias, der schon seit dem Tod des langjährigen Parteiführers Ezekias Papaioannou 1988 Generalsekretär von AKEL war, wurde wieder zum Präsidenten des Hohen Hauses gewählt; vgl. http://www.parliament.cy/parliamentgr/003_01_02.htm (auf Griechisch) (Abruf am 10. Mai 2009).

76 Vgl. Dimitrios Parashu, a.a.O. (Fn. 4).

77 Vgl. ebenda.

78 Vgl. ebenda. Zum Abgeordneten der armenischen Minderheit wurde erstmals Vartkes Mahdessian, für die Maroniten Antonios Hatziroussos und für die Latiner Benito Mantovani von den betreffen- 
Nach seiner erneuten Wahl zum Parlamentspräsidenten blieb Dimitris Christofias bis zu seiner Wahl zum Staatspräsidenten Zyperns Ende Februar 2008 im Amt, gehört aber seit diesem Zeitpunkt gemäß Art. 41 Abs. 1 (wie 1977 im Fall von Spyros Kyprianou) nicht mehr dem Hohen Haus als Mitglied an. Seither ist Marios Garoyian (DIKO) Chef des Repräsentantenhauses. Die Koalitionsparteien AKEL, DIKO und EDEK hoffen im Zuge der aktuell wieder aufgenommenen Verhandlungen zur Überwindung des Zypernproblems eine bikommunale und bizonale Föderalrepublik auf der Insel schaffen zu können. Die Opposition ist in der Sache zwar damit einverstanden, fürchtet wohl aber, dass Christofias zu weit gehende Zugeständnisse an die türkischen Zyprer macht.

Bei der letzten Parlamentswahl kam es, wenn auch erst im Zuge der Entscheidung „Ibrahim Aziz vs. Republic of Cyprus“ des Europäischen Gerichtshofes für Menschenrech$\mathrm{te}^{79}$, erstmals zur Wahrnehmung des aktiven wie vereinzelt auch passiven Wahlrechts von in der Republik Zypern wohnhaften türkischen Zyprern, auf Basis des Notrechts - immerhin ein kleiner Hoffnungsschimmer, auch wenn (oder, besser, gerade weil) dies nicht dem Wortlaut und Sinn der Verfassung von 1960 entspricht ${ }^{80}$.

\section{Geänderte Verfassung für ein geeintes Zypern}

Das Repräsentantenhaus Zyperns hat, insbesondere nach 1974, eine für die engere Region erstaunliche Konstanz entwickeln können. Dies geschah unabhängig sowohl von den genuinen Dysfunktionalitäten der seinerzeit hauptsächlich von britischer Seite unterstützten und „auferlegten“ Verfassung als auch von der stets präsenten Teilung der Insel (und den damit verbundenen Widrigkeiten in der Verfassungsumsetzung). Legislative und Exekutive arbeiten zumeist harmonisch zusammen, ohne dass im Regelfall eine Regierung trotz ihres verfassungsrechtlich geregelten relativen Vorteils ein Repräsentantenhaus „an die Wand spielen“ würde. Auch die Parteien tragen zur Konstanz bei, denn Schwankungen bei den Wählerstimmen hielten sich in den letzten Jahrzehnten in Grenzen.

Gemäß der verfassungsrechtlich vorgeschriebenen fünfährigen Dauer der Legislaturperiode findet die nächste Wahl zum Repräsentantenhaus Zyperns im Jahr 2011 statt. Vor dem Hintergrund der Wahlversprechen des neuen Staatsoberhauptes Christofias ${ }^{81}$, sich in erneuten Gesprächen um eine weitergehende Annäherung zum Norden der Insel und eine eventuelle Wiedervereinigung Zyperns zu bemühen ${ }^{82}$, und seiner Treffen mit Repräsentan-

den Bevölkerungsgruppen wiedergewählt; vgl. http://www.parliament.cy/parliamentgr/002_ 10_11.htm (auf Griechisch) (Abruf am 12. Mai 2009).

79 Entscheidung vom 22. Juni 2004; vgl. Achilleus Aimilianidis, a.a.O (Fn. 9), S. 149.

80 Art. 63 Abs. 2 der Verfassung sieht vor, dass griechische und türkische Zyprer nur in getrennten Wahllisten aufzuführen sind; vgl. Achilleus Aimilianidis, a.a.O (Fn. 9), S. 150. Diese Wahrnehmung des aktiven wie passiven Wahlrechts wurde bereits teilweise im Rahmen der Europawahlen von 2004 auf Zypern in der Praxis angewandt.

81 Wie im Fall des Rücktritts von Kliridis als Parteivorsitzender von DISY nach seiner Wahl zum Staatspräsidenten 1993 (Nachfolger wurde Yannakis Matsis; vgl. Dimitrios Parashu, a.a.O. (Fn. 4)) gab auch Christofias Anfang 2009 das Amt des Parteiführers von AKEL auf; sein Nachfolger ist Andros Kyprianou, vgl. den entsprechenden Bericht der Zeitung Kathimerini vom 22. Januar 2009 (auf Griechisch).

82 Vgl. entsprechende Artikel der griechischen Zeitung Eleftherotypia vom 24. Februar 2008 (auf Griechisch). 
ten der türkischen Zyprer ${ }^{83}$ bleibt abzuwarten ${ }^{84}$, ob dann die Sitze der türkisch-zyprischen Abgeordneten erstmals seit 1963 wieder besetzt sein werden. Sollte dies der Fall sein, sollten diejenigen Punkte aus Alt-Staatspräsident Makarios' Vorschlag zur Änderung der Verfassung übernommen werden, die das Repräsentantenhaus direkt betrafen: (1) die Wahl des Präsidenten des Hohen Hauses und seines Vizepräsidenten durch alle gesetzlichen Mitglieder, Zyperngriechen und -türken; (2) die effektive Möglichkeit der Vertretung des griechisch-zyprischen Parlamentspräsidenten durch seinen türkisch-zyprischen Vize; (3) die Abschaffung der nach Bevölkerungsgruppen getrennten Abstimmungen im Repräsentantenhaus, in welchem Artikel der Verfassung sie auch vorgesehen sind ${ }^{85}$. Nur auf diese Art und Weise könnte eine effektive Zusammenarbeit im Hohen Haus eines einheitlichen Zypern funktionieren.

83 Vgl. unter anderem den Bericht der Zeitung Kathimerini vom 12. September 2008 (auf Griechisch); relevante Berichte der Gesellschaft für Zypernstudien vom 12. Oktober 2008 („Wöchentliche Treffen zu Zypern-Gesprächen vereinbart“) und 27. Oktober 2008 („Sechste Runde der Zyperngespräche“) auf http://www.cypern.de/?m=200810 (Abruf am 15. Dezember 2008) sowie die Einschätzung eines nur relativen Fortschritts in der Zeitung Kathimerini vom 18. Dezember 2008 (auf Griechisch) und Christofias' erklärtes Bestreben, die Gespräche kontinuierlich und effektiv fortzuführen, vgl. das entsprechende Grußwort von Christofias in der Zeitung Charavgivom 25. Dezember 2008 (auf Griechisch).

84 Eine zusätzliche Schwierigkeit dürfte wohl nach dem 19. April 2009 und der nunmehr effektiven Zwangsmachtteilung zwischen Sozialdemokraten und Nationalkonservativen im türkisch besetzten Norden Zyperns entstehen; vgl. unter anderem den entsprechenden Bericht der Zeitung Kathimerini vom 20. April 2009 (auf Griechisch).

85 Vgl. in diesem Zusammenhang Jörg-Detlef Kühne, a.a.O. (Fn. 8), S. 80; Iossif Iossif, a.a.O. (Fn. 2), S. 73; Konstantinos Goumas / Stelios Kotsiopoulos (Hrsg.), a.a.O. (Fn. 2), S. 980.

\title{
Die Verurteilung des Premierministers durch Urteil des thailändischen Obersten Gerichtshofs vom 21. Oktober 2008
}

\author{
Natjira Kanthong
}

Vorbemerkung der Redaktion: Das nachstehend wiedergegebene Urteil gegen den Vorgänger des jetzigen thailändischen Premiers verdient in zweifacher Hinsicht Aufmerksamkeit: Erstens politisch, weil es durchaus nicht zu einer wirklichen Beruhigung der innenpolitisch höchst angespannten Lage dort beitragen konnte. Vielmehr scheint es zur Zeit nach den politischen Dauerdemonstrationen in Bangkok nur um eine gewisse Verschnaufpause in dem Bemühen der dem Vorgänger verpflichteten so genannten Rothemden zu gehen, ihn wieder an die Macht zu bringen. Dabei bietet das vorliegende Gerichtsverfahren nur eine Facette der politisch-sozialen Transformation, in der sich Thailand nicht anders als etliche seiner Nachbarländer befindet. Begleitet von Vorwürfen der Vetternwirtschaft und des Stimmenkaufs, geht es um eine langsame Verschiebung von den angestammten alten Eliten 